\title{
DAYEM-MARTIN (SIS TUNNEL JUNCTION) MIXERS FOR LOW NOISE HETERODYNE RECEIVERS
}

\author{
T. G. Phillips and D. P. Woody \\ California Institute of Technology, Pasadena, CA 91125 \\ and \\ G. J. Dolan and R. E. Miller \\ Bell Labs, Murray Hil1, N. J. 07974 \\ and \\ R. A. Linke \\ Bel1 Labs, Crawford Hi11, N. J. 07733
}

Superconducting thin film tunnel junctions of sma11 area $\left(\cdot 1 \rightarrow 1 \mu \mathrm{m}^{2}\right)$ have properties which make them suitable for high frequency $(\geqslant 100 \mathrm{GHz})$ heterodyne receivers. Both pair and single quasiparticle tunneling is present in these devices, but it is found that the mixing due to the pair effect is apparently excessively noisy, whereas the single quasiparticle effect has a low noise character which gives hope for near quantum limited performance. The physical effect involved is photon assisted quasiparticle tunneling and was first observed by Dayem and Martin. ${ }^{1}$ We have made laboratory tests at 115 and $230 \mathrm{GHz}$ which gave single side band (SSB) mixer noise temperatures of 60 and $300 \mathrm{~K}$ respectively. Also we have fabricated a 90-140 GHz receiver for the Caltech Owens Valley Radio Observatory which has an overall receiver noise temperature of about $300 \mathrm{~K}$ (SSB).

\section{Introduction}

\section{Mi11imeter and Submi11imeter Astronomical Lines}

The region of the electromagnetic spectrum from a few $\mathrm{GHz}$ to a few thousand $\mathrm{GHz}$ contains many interesting narrow line transitions of both atoms and molecules. Many species of atoms (e.g. $H, C, 0)$ and molecules (e.g. $\mathrm{H}_{2}, \mathrm{CO}$ ) exist in the clouds of gas within our galaxy, and studies of these species provide understanding of both the physics and chemistry of this interstellar gas which, of course, provides the material for forming stars. Typical temperatures for the dense interstellar clouds range from 5 to $100 \mathrm{~K}$ so that energy levels can be populated for both fine structure transitions of atoms and rotational transitions of molecules.

Densities in the interstellar medium are low by laboratory standards, usually in the range $10^{3}-10^{6}$ $\mathrm{cm}^{-3}$ for molecular clouds. So pressure broadening effects are negligible and linewidths are determined by Doppler effects caused, for instance, by turbulence over large regions of the clouds. Linewidths, therefore, are small and spectral resolution of $10^{-6}$ or better may be required.

\section{Heterodyne Receivers}

To achieve high spectral resolution it is usual in the radio, millimeter and submillimeter-wave bands to employ heterodyne receivers in which a local oscillator wave is coherently mixed with the signal. The mixer element is usually a diode type device providing non linear response. The device must be capable of carrying currents at the signal frequency. The difference frequency between the signal and the local oscillator is available as an intermediate frequency (IF) over a somewhat restricted range (usually less than a $(\mathrm{GHz}$ ) depending on the capability of the low noise IF amplifier. Such a receiver is characterized by a noise temperature $\left(T_{R}\right)$ which is contributed to by the noise generated in the mixer, which is equal to or greater than the quantum noise due to fluctuations in the local oscillator power, plus the noise of the IF amplification chain which appears as effectively multiplied by the power conversion loss factor (L) of the mixer.

$$
T_{R}=T_{M}+L T_{I F}
$$

For the purposes of this paper al1 numbers are quoted for single side band operation (SSB), which is the usual spectroscopic mode. Noise temperature measurements are usually made with white noise, fixed temperature, loads applied to the front end of the receiver, which affect both side bands equally. For small values of the IF it is often the case that the double side band values are one half the SSB values, since both side bands convert power equally. In principle power loss to the converted side band can be avoided (image suppression) by differential matching if the IF is large enough, or by use of a two element front end. At high frequencies these techniques are not usually practicable.

Spectroscopy is performed by dividing the IF band into channels of width $\Delta \nu$ and rectifying and integrating the noise in each channel separately. A11 mixer receivers operating in the Rayleigh-Jeans limit $\left(h \nu<k T_{R}\right.$ ) provide a signal to noise ratio given by the Dicke radiometer equation:

$$
S / N=\frac{T_{S}}{T_{R}} \sqrt{\Delta v \Delta t}
$$

where $T_{S}$ is the equivalent black body temperature of the signal and $t$ is the integration time. Clearly it is very important to reduce $T_{R}$ to a minimum.

A11 radiometric devices are governed by equation (2), however not all are classical diode mixers. Quantum mixers, discussed below, and bolometer mixers, not discussed in this article, have properties which make them suitable for certain purposes. Figure $I$ shows a graph for measured mixer noise temperatures $\left(T_{M}, S S B\right)$, equation (1), for various devices at frequencies up to $\sim 800 \mathrm{GHz}$.

The lowest noise temperatures at high frequencies are found for InSb bolometer mixers. These are buTk, single crystal devices, which do not suffer from embedding network difficulties (i.e. they have easily computed resistive and reactive impedance components in a waveguide mount), however they have very limited If bandwidths due to the intrinsic electronic relaxation time of the material.

\section{Superconducting Mixers}

The lowest noise temperatures in the $10-100 \mathrm{GHz}$ range are found for superconducting mixer devices. This is primarily because the smali value of the superconducting gap $(\sim 1 \mathrm{mV}$ rather than $\sim T V$ for a semiconductor) produces only a small current flow at typical 


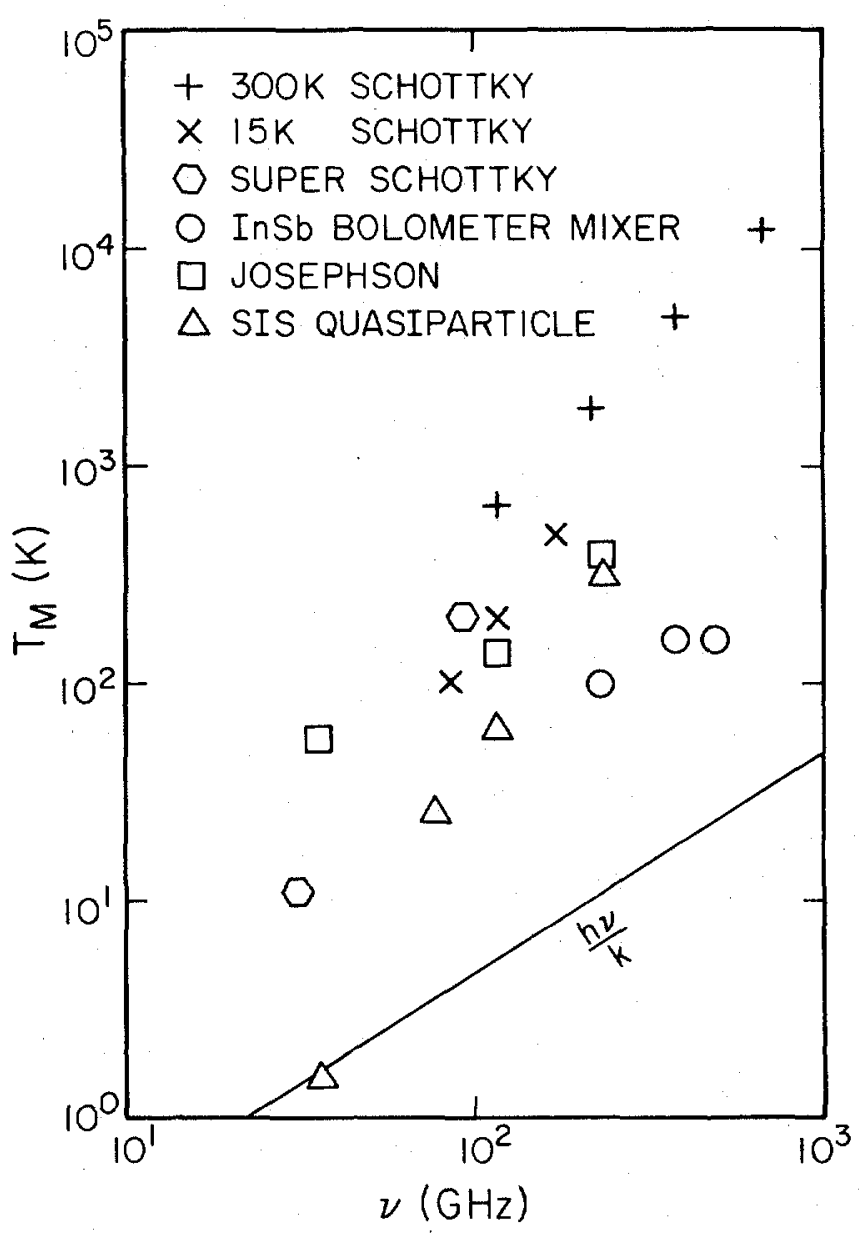

Fig. 1. SSB mixer noise temperatures for several different types of millimeter and submillimeter heterodyne receivers. Data for $300 \mathrm{k}$ Schottky mixers are from references $[2-5]$. Data for $15 k$ Schottky mixers are from references [6-8]. Data for super Schottky mixers are from references [9 and 10]. Data for InSb bolometer mixers are from reference [11]. Data for Josephson junction mixers are from references [12-14]. Data for SIS quasiparticle mixing are from $[15-17]$.

impedances of $\sim 100 \Omega$, so reducing shot noise in the device. Also, the onset of current at the gap bias often takes place in a voltage range < hu/e so that quantum effects are important. In principle all devices could have quantum noise limited performance, but in practice it is difficult to reduce unwanted currents (not associated with local oscillator induced effects) to zero. A further advantage of superconducting mixer devices is that they may show conversion gain rather than loss, so eliminating receiver noise due to If amplifier noise.

From equation (1) it is clear that one only has two aspects of the device to optimize, the mixer noise and the conversion efficiency. However, some types of device, which at first seemed promising, have been eliminated due to difficulties in one of these areas. An example is the Josephson effect mixer which has persistently exhibited an excess noise phenomenon. This will be clearly seen in the data below.
By contrast we find ${ }^{18}$, as do others ${ }^{19}$, that the mixing effect associated with single quasiparticle tunneling exhibits noise which is largely local oscillator photon noise, with some extra contribution from shot noise due to certain unwanted currents. These 'DayemMartin' effect devices are likely to prove most effective for radioas tronomy.

At high frequencies ( $>100 \mathrm{GHz}$ ) the conversion efficiency is reduced from the theoretical value by parasitic impedances in the microwave circuit. The traditional technique for mounting diodes in a waveguide involves contacting the diode with a wire spring or 'whisker', running along the waveguide electric field direction. This is avoided in thin film superconducting mixers since the whole device is formed lithographically on a substrate which can contain the contact leads and IF choke structure (see Figure 2). The device designer then has control over the microwave circuit, which should allow the reduction of conversion losses due to parasitics.

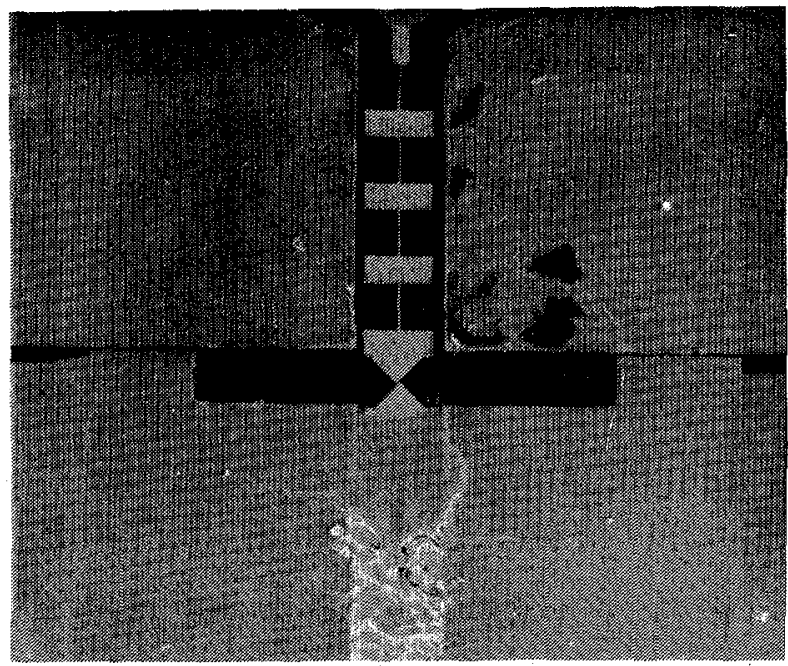

Fig. 2. Substrate containing an SIS junction with dc and IF connection and RF choke structure mounted across a $1 / 4$ height. WR- 8 waveguide.

Finally, of great importance in the development of. superconducting mixer devices, there now exists a quantum mechanical theory due to Tucker 20 which predicts the performance of Dayem-Martin mixers. This theory allows comparison with experiment over a range of conditions; e.g. the mixing may be examined for various values of bias such that single or multiple photon effects come into play. This flexibility should allow the device designer to separate out the parasitic effects from those intrinsic to the device.

\section{Quasiparticle Tunneling Mixer Devices}

\section{Types of Devices}

At the present there appear to be three types of device which exhibit quasiparticle current mixing. The first to be investigated was the Super-Schottky Diode 21 . Basically this only differs from the Schottky diode by replacement of the normal metal electrode by a superconductor and by the increase of semiconductor dopants to give a narrower depletion 
layer suitable for use as a tunneling barrier. As a microwave device it suffers from the problems of whisker contacts.

A second type is the SIN structure. Here the tunnel junction is a thin film structure with one nor$\mathrm{ma} 1$ and one superconducting electrode. An early attempt to utilize these devices as microwave detectors $^{22}$ was unsuccessful, in part due to metallurgical difficulties. However, for reasons that will appear below, at frequencies $>300 \mathrm{GHz}$ it may be that these devices will be required.

The third, and currently most popular structure, is the SIS device. Initial uses of these at $115 \mathrm{GHz}^{18}$ and $36 \mathrm{GHz}{ }^{19}$ showed efficient mixing, low noise temperatures and confirmed that quasiparticle currents were involved. Subsequently it has become clear that the photon assisted tunneling observed by Dayem and Martin $^{1}$ and explained by Tien and Gordon ${ }^{23}$ is the dominant feature of the device. This effect is manifest as steps in the I- $V$ characteristic when local oscillator power is applied, spaced $\Delta V=h v / e$ from the gap structure. It is easily seen in the SIS system because there are two sharp density of states structures convolved, compared with just one for the SIN or Super Schottky devices. SIS devices also show Josephson steps, of course, spaced $\Delta V=h \nu / 2 e$ from zero bias.

Both SIS and SIN structures can be formed as arrays of devices and at least two investigations have been reported. 16,18

\section{Construction of Devices}

In our investigation junctions fabricated by the methods of Dolan ${ }^{18}$ have been employed. Electron beam lithography is used to generate masks from which photoresist bridge structures are made. An example of such a bridge structure is shown in figure 3 . Figure 4

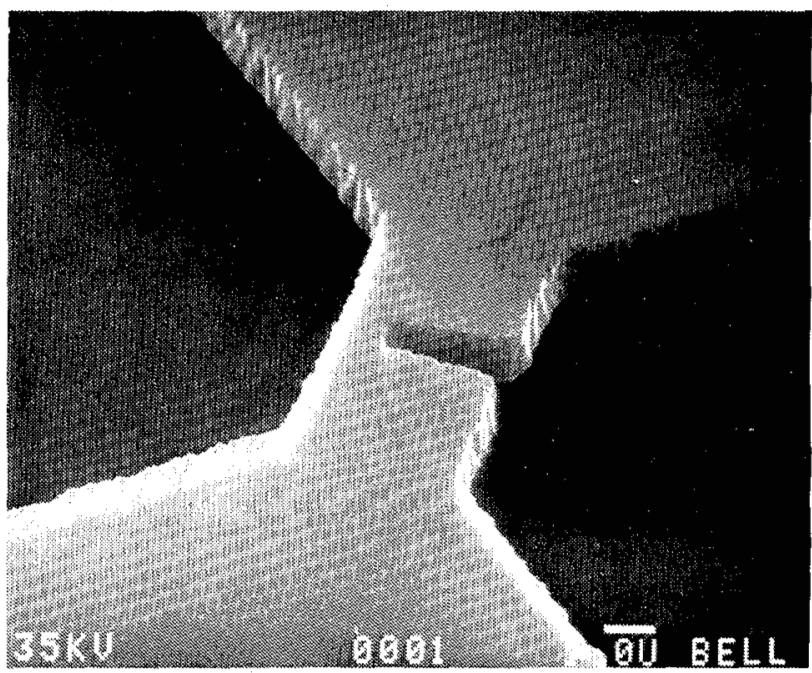

Fig. 3. Scanning electron microscope picture of a suspended photoresist bridge structure used to fabricate small area junctions
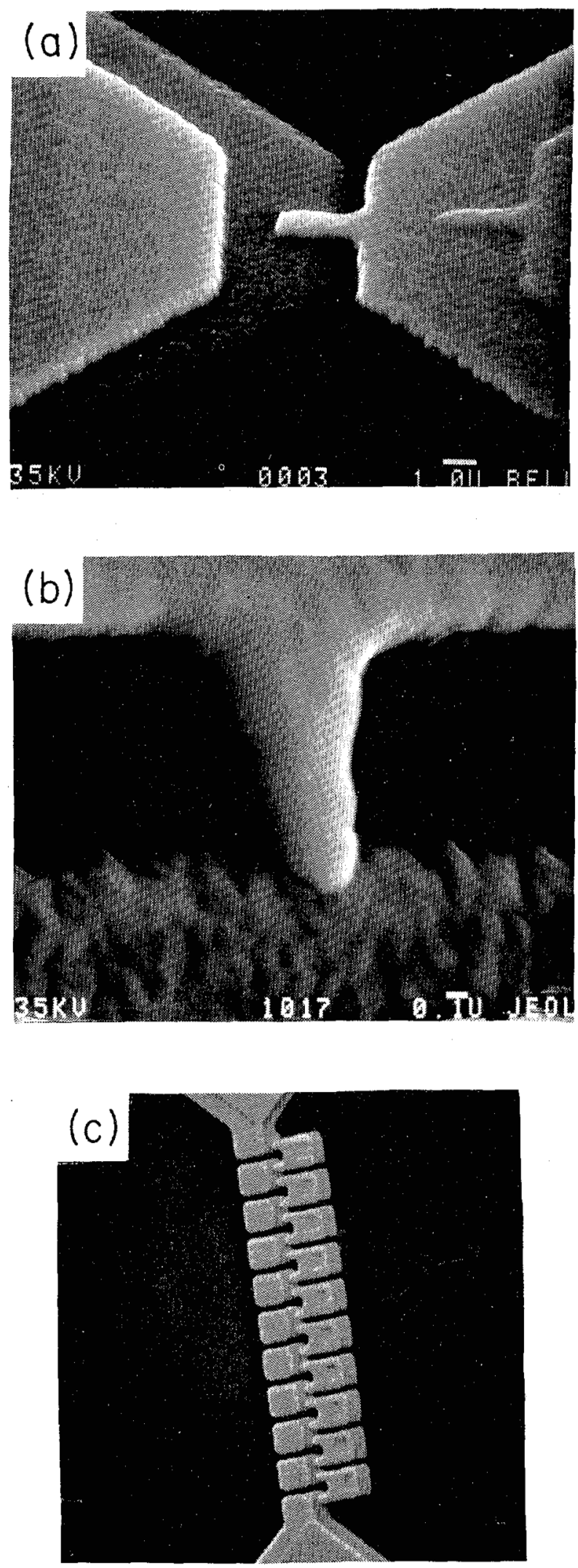

Fig. 4. (a) An SIS junction made using the photoresist structure shown in Fig. 3. (b) An SIS junction whose area is near the lower limit achievable by this technique. (c) A series array of 20 SIS junctions. 
shows examples of evaporated structures made with this technique. By evaporating past the bridge at different angles, overlapping structures are formed. Both very small area junctions $\left.(\sim \cdot]_{\mu} \mathrm{m}^{2}\right)$ and arrays of junctions can be made in this way.

\section{Tunneling and Mixing Characteristics}

\section{Results at $115 \mathrm{GHz}$.}

I- $V$ characteristics for an SIS device, with $\mathrm{Pb}+$ $4 \%$ in electrodes, are shown in figure $5 \mathrm{a}$. The photon assisted tunneling steps become clearly visible with increasing local oscillator (LO) power. Two steps are observable above and below the gap bias voltage, but bel ow about $1.5 \mathrm{mV}$ bias the characteristics are confused by the presence of Josephson currents.

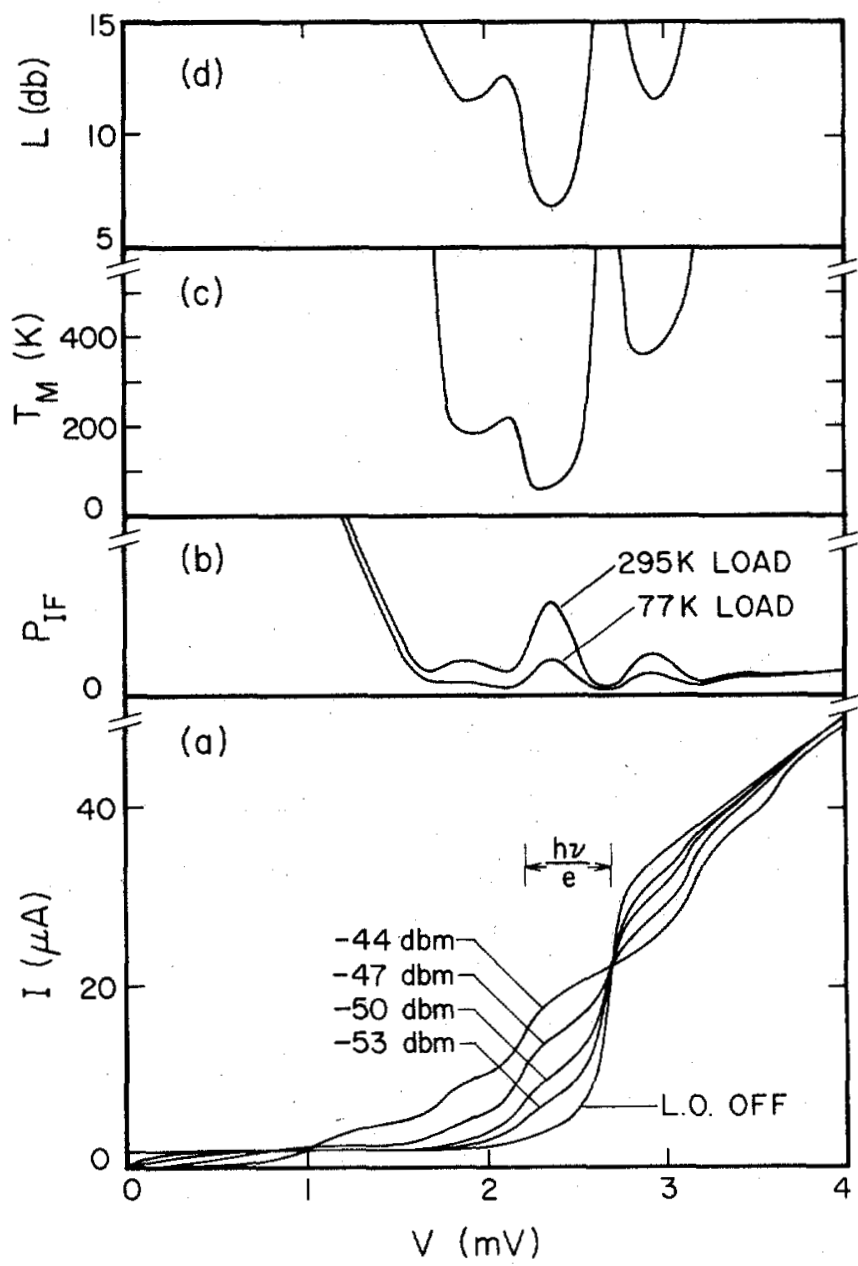

Fig. 5. (a) Current vs. voltage characteristics for as SIS junction with different $115 \mathrm{GHz}$ LO power levels applied. (b) IF output power with hot and cold load signals applied. (c) SSB mixer noise temperature. (d) SSB mixer conversion 1oss.

Mixing response is indicated in figure $5 \mathrm{~b}$. For an Lo power of about $10^{-8}$ watts the sensitivity is roughly optimum as can be seen from the curves of $5 \mathrm{a}$. When the waveguide is coupled to an LO source of that power and also to a black body load (by a means described in the receiver description below) the down converted power coupled to the IF is a function of bias voltage, as displayed in 5b. Knowing the IF amplifier noise temperature and two black body load temperatures allows a measurement, at each bias point, of the mixer temperature and conversion loss, using a somewhat more sophisticated version of equation (1). These quantities are plotted in figures $5 c$ and $d$, and are SSB values. For this particular junction the peak responsivity, minimum noise temperature and optimum conversion loss a11 occur on the single photon assisted tunneling step below the gap. Some of our junctions have shown slightly improved performance on the second step. The best values for $T_{M}$ and $L$ found at $115 \mathrm{GHz}$ have been about $60 \mathrm{~K}$ and $7 \mathrm{~dB}$ respectively.

It is noticeable in figures $5 b, c$ and $d$ that the noise increases dramatically and the system performance worsens in the region at low bias where the Josephson currents are dominant. This effect is more detrimental at higher frequencies.

\section{Results at $230 \mathrm{GHz}$}

Measurements at $230 \mathrm{GHz}$ were carried out on the same type of junction as used at $115 \mathrm{GHz}$. The waveguide arrangement was somewhat simplified because of the greater construction difficulties at such frequencies. In fact a circular fundamental waveguide mode was used, rather than the rectangular reduced $(1 / 4)$ height system employed at $115 \mathrm{GHz}$. Figure 6a shows the I-V characteristics with and without Lo power, the only significant change being the doubling of the Dayem-Martin step width. The region below $1.5 \mathrm{mV}$, for which the Josephson currents are dominant in these junctions, now protrudes almost into the region of the first step. Figure $6 b$ shows the dramatic increase in IF noise at that voltage. We have attempted to deal

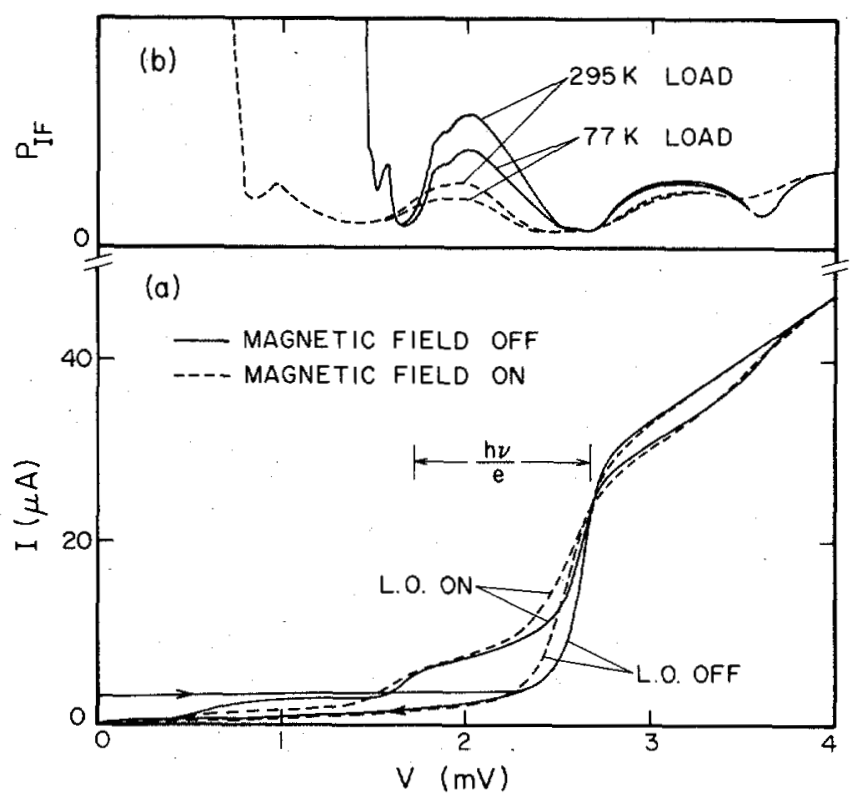

Fig. 6. (a) Current vs. voltage characteristics for a junction with and without $230 \mathrm{GHz}$ LO power applied. (b) IF output power with hot and cold load signals applied. The effect of applying a magnetic field is shown by the dashed lines. 
with the problem by applying a magnetic field to the junction. This has the effect of reducing the voltage range in which the junction shows hysteresis. The dashed curve of 6 a shows negligible hysteres is compared with the curve for no magnetic field. As anticipated, 6b shows a reduced range for the excess noise. However, in these particular experiments the field on the junction is not sufficiently homogeneous and has smeared the gap (6a), resulting in reduced mixing sensitivity (6b). It can be remarked here that a series array of junctions may allow a way out of this problem. A disadvantage for arrays at high frequency is that the series inductance between junctions must be kept smal1, which accounts for the complicated structure of figure $4 \mathrm{c}$. A complete solution to the Josephson current problem would be to use SIN devices, of course.

Our best single side band performance to date for SIS devices at $230 \mathrm{GHz}$ is $\mathrm{T}_{M} \leqslant 300 \mathrm{~K}$. As yet, we have not accurately measured the conversion loss at this higher frequency.

\section{0-140 GHz Astronomical Receiver}

A receiver to mount at the focus of a $10 \mathrm{~m}$ telescope at Caltech's Owens Valley Radio Observatory, using an SIS mixer element, is now nearing completion. A simplified block diagram of the receiver is shown in figure 7 . This figure also demonstrates the method of measurement of the receiver and mixer performance parameters. The parts within the box are in vacuum, bolted to the cold plate of a liquid helium cryostat. Some measurements have been made at $4.2 k$, but the best results are generally found at lower temperatures.

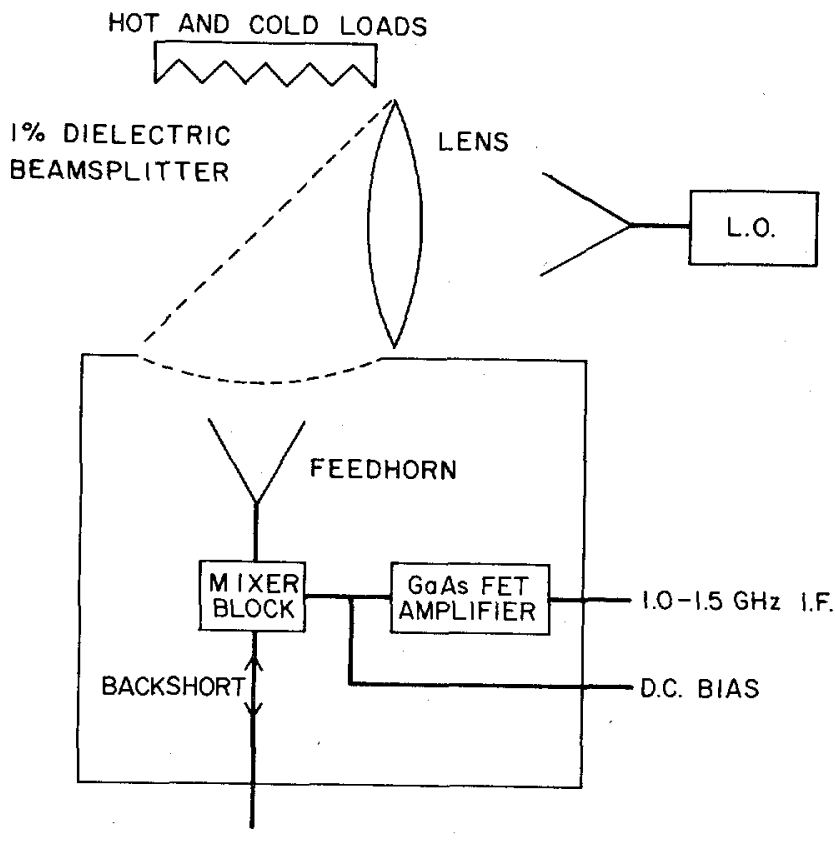

Fig. 7. Schematic diagram of $115 \mathrm{GHz}$ receiver.

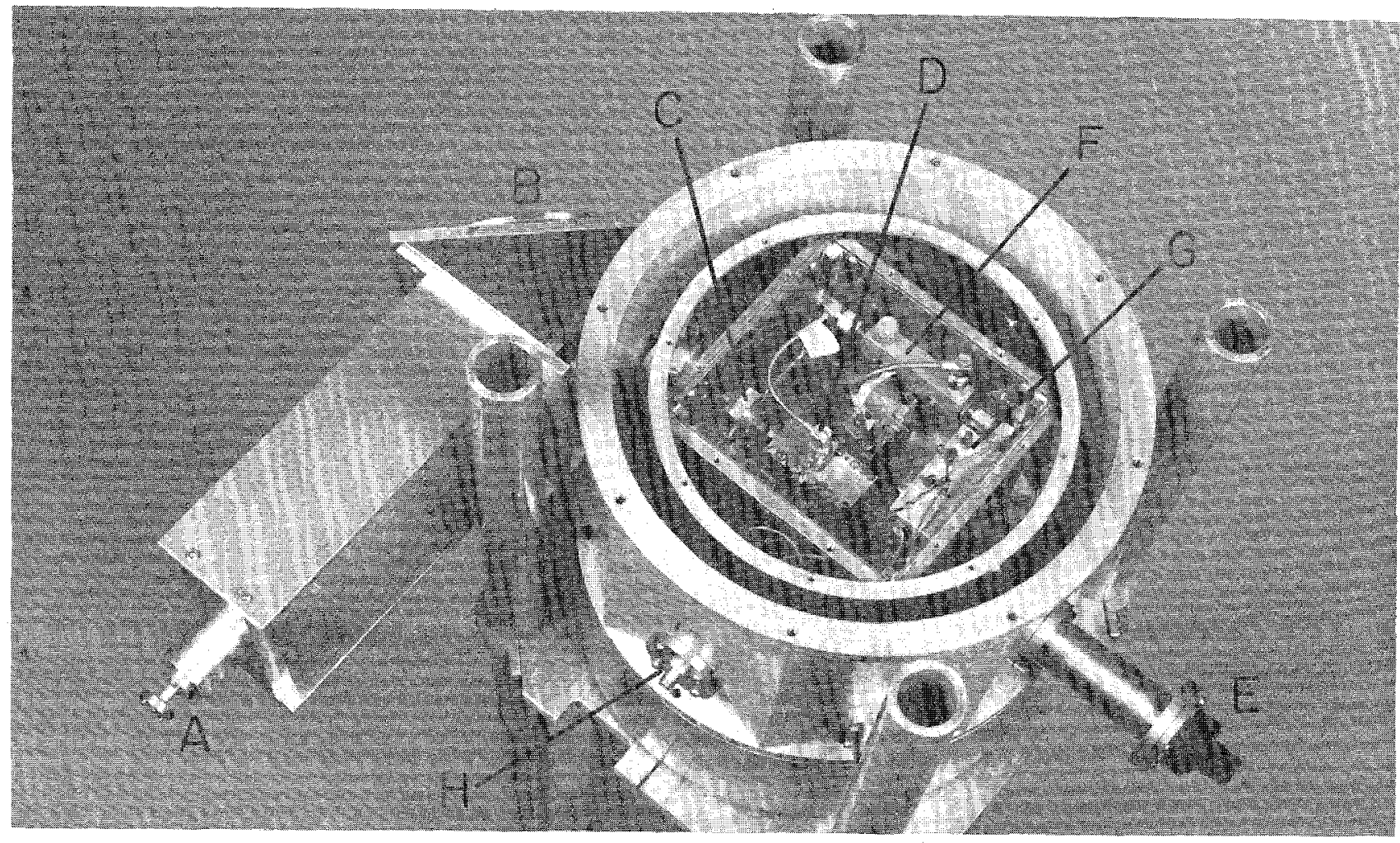

Fig. 8. Picture of $115 \mathrm{GHz}$ receiver to be used for astronomical observations. The principal elements are; A, LO feedhorn; B, 1\% dielectric beamsplitter; $C$, mixer feedhorn; D, mixer block; E, backshort tuner; $F, 30 \mathrm{db}$ directional coupler for coupling test signals into the IF line; $G, 1.4 \mathrm{GHz}$ cooled GaAs FET IF amplifier; $H$, IF output port. 
The dielectric beam splitter allows the signal to pass freely and couples in only $\sim 7 \%$ of the Lo power. This is possible because of the low LO power requirement for the SIS mixer. Figure 8 is a photograph of the receiver dewar with radiation shields removed.

A prototype version of this receiver, built at Bell Labs, was used in June 1979 to determine its suitability for astronomical operation. No major problems were encountered. The table below indicates performance figures for the prototype and current receiver. The current receiver has a system temperature of $300 \mathrm{~K}$ at $115 \mathrm{GHz}$, which is comparable with or better than contemporary cooled Schottky diode receivers. The table also indicates what would result if the best measured individual component figures could be achieved in the final configuration. This is a matter of selection and correct matching. Finally, the theory column shows the expectations from the theory of Tucker. 20

Table 1

SIS DAYEM-MARTIN RECEIVER AT $115 \mathrm{GHz}$

$\begin{array}{lcccc} & \begin{array}{c}\text { ACHIEVED ON } \\ \text { TELESCOPE } \\ \text { JUNE 1979 } \\ \text { (B.T.L.) }\end{array} & \begin{array}{c}\text { LAB } \\ \text { (C.I.T.) }\end{array} & \begin{array}{c}\text { BEST } \\ \text { ACHIEVED } \\ \text { (C.I.T. } \\ \text { B.T.L.) }\end{array} & \text { THEORY } \\ T_{\text {IF }} & 60 \mathrm{~K} & 15 \mathrm{~K} & 11 \mathrm{~K} & <10 \mathrm{~K} \\ L_{S S B} & 8 \mathrm{db} & 8.5 \mathrm{db} & 6 \mathrm{db} & \text { GAIN } \\ T_{M, S S B} & 95 \mathrm{~K} & 180 \mathrm{~K} & 60 \mathrm{~K} & <15 \mathrm{~K} \\ T_{\text {REC, SSB }} & 400 \mathrm{~K} & 300 \mathrm{~K} & (105 \mathrm{~K}) & <30 \mathrm{~K}\end{array}$

\section{Acknowledgements}

Initial aspects of this work were carried out in collaborations with R. C. Dynes and J. H. Magerlein. Conversations with T. A. Fulton, J. M. Rowell and T. C. L. G. Sollner have been most helpful. Work at Caltech was carried out with support from NSF grants \# AST-76-1334 and AST 80-07645.

\section{References}

1. A. H. Dayem and R. J. Martin, "Quantum Interaction of Microwave Radiation with Tunneling Between Superconductors," Phys. Rev. Lett., 8, 246 (1962).

2. A. R. Kerr, "Low-Noise Room Temperature and Cryogenic Mixers for 80-120 GHz," IEEE Trans. Microwave Theory Tech., MTT-23, 781 (1975).

3. E. R. Carlson, M. V. Schneider and T. F. McMaster, "Subharmonically Pumped Millimeter-Wave Mixers," IEEE Trans. Microwave Theory Tech., MTT-26, 706 (1978).

4. N. R. Erickson, "A $0.9 \mathrm{~mm}$ Heterodyne Receiver for Astronomical Observations," IEEE International Microwave Symp. MTT-S, 438 (1978).
5. M. R. Fetterman, P. E. Tannenwald, B. J. Clifton, C. D. Parker, W. D. Fitzgerald and N. R. Erickson, "Far IR Heterodyne Radiometric Measurements with Quasi-Optical Schottky Diode Mixers," App1. Phys. Lett. 33, 151 (1978).

6. E. Kolberg, "Schottky Barrier Diode Mixers," URS I Symp. on Millimeter Technology in Radioastronomy, Grenoble (1980).

7. R. A. Linke, M. V. Schneider and A. Y. Cho, "Cryogenic Millimeter-Wave Receivers Using Molecular Beam Epitaxy Diodes," IEEE Trans. Microwave Theory and Tech. MTT $-26,935$ (1978).

8. S. Weinreb and J. Payne, Personal communication.

9. M. McCo11, M. F. Bottjer, A. B. Chase, R. J. Pederson, A. H. Silver and J. R. Tucker, "The Super-Schottky Diode at $30 \mathrm{GHz}$," IEEE Trans. Magn. MAG-15, 468 (1979).

10. W. Wilson, Personal communication.

11. T. G. Phillips, Unpublished.

12. Y. Taur, J. H. Classen and P. L. Richards, "Josephson Junctions as Heterodyne Detectors," IEEE Trans. Microwave Theory Tech. MTT-22, 1005 (1976).

13. Y. Taur and A. R. Kerr, "Low Noise Josephson Mixers at $115 \mathrm{GHz}$ Using Recyclable Point Contacts," App1. Phys. Lett., 32, 775 (1978).

14. Th. de Graauw, Personal communication.

15. T. M. Shen, P. L. Richards, R. E. Harris and F. L. Lloyd, "Conversion Gain in MM-Wave Quasiparticle Heterodyne Mixers," App1. Phys. Lett. 36, 777 (1980).

16. S. Rudner, M. J. Feldman, E. Kolberg and T. Claeson, "SIS Quasiparticle Mixing with Long Antenna Coupled Arrays," Proc. I. C. Squid II Conf., 1980.

17. This work.

18. G. J. Dolan, T. G. Phillips and D. P. Woody, "Low Noise $175 \mathrm{GHz}$ Mixing in Superconducting 0xideBarrier Tunnel Junctions," Appl. Phys. Lett. 34 , 347 (1979).

19. P. L. Richards, T. M. Shen, R. E. Harris and F. L. Lloyd, "Quasiparticle Heterodyne Mixing in SIS Tunnel Junctions," Appl. Phys. Lett. 34, 345 (1979).

20. J. R. Tucker, "Quantum Limited Detection in Tunnel Junction Mixers," IEEE J. Quantum Electron. QE-15, 1234 (1979).

21. M. Mc Col1, M. J. Millea and A. H. Silver, "The Superconductor-Semiconductor Schottky Barrier. Diode Detector," App 1. Phys. Lett., 23, 263 (1973).

22. T. G. Phillips, R. C. Dynes and J. M. Magerlein, Unpublished.

23. P. K. Tien and J. P. Gordon, "Multiphoton Process Observed in the Interaction of Microwave Fields with the Tunneling between Superconducting Films," Phys. Rev. 129, 647 (1963). 